\section{Tying up loose ends}

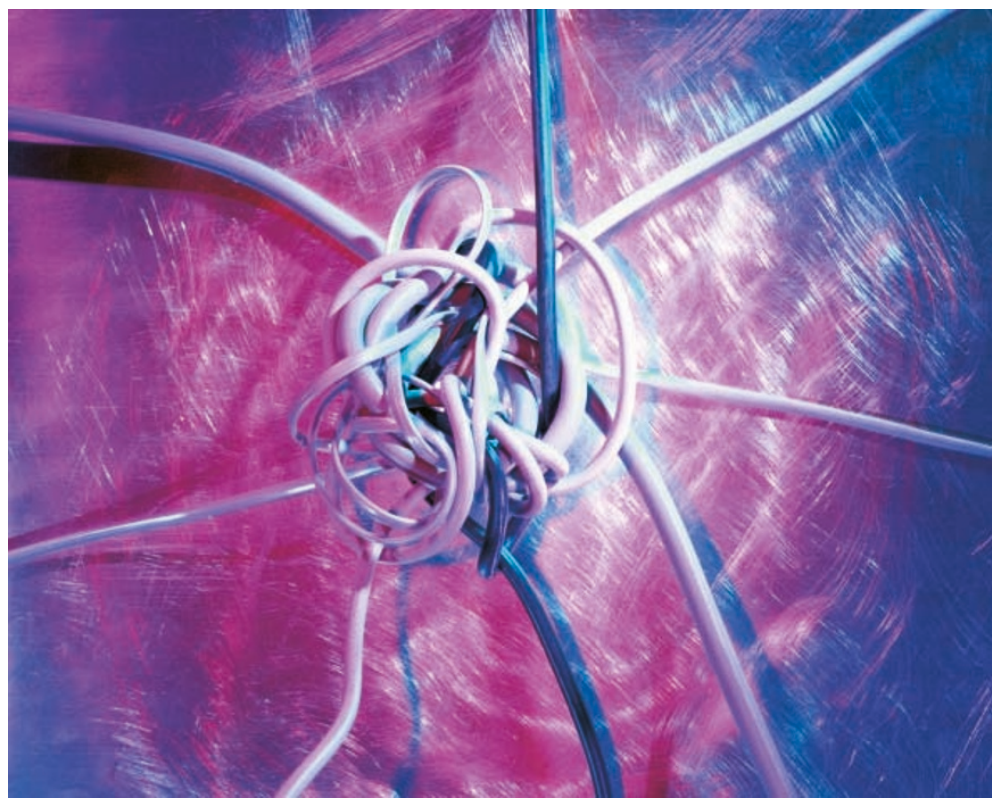

It's always a bonus for researchers when apparently separate pathways for pathogenetic mechanisms in fact conveniently and neatly intertwine. A report by Stuart Lipton and colleagues in Science shows how two proposed mechanisms for nerve-cell death are intimately linked, and should therefore help to direct therapies aimed at treating neurological disorders.

It had been known that the nervecell damage that triggers their demise during stroke, Alzheimer's disease and other neurodegenerative diseases not only occurs from inside the cell but externally too. The best-characterized nerve-cell-death pathways occur inside cells, but in terms of external mechanisms, two observations had previously been made - levels of matrix metalloproteinases (particularly MMP9) are elevated in neurodegenerative disorders, and nitric oxide (NO) can modulate the activity of proteins (in a manner that can be described as being analogous to phosphorylation) by reacting with cysteine thiols to form an S-nitrosylated derivative.

Now, Lipton and colleagues show that these two events are linked. They found that NO switches on the overexpression of MMP enzymes, which, in turn, chew up the environment that surrounds nerve cells.

Initial in vitro analysis showed that NO can directly activate MMP9 and induce neuronal apoptosis. To confirm their results in vivo, Lipton and colleagues used mass spectrometry to characterize the events during focal ischaemia (the lack of blood supply owing to the occlusion of an artery) and reperfusion (the return of blood to an ischaemic region, which is also accompanied by tissue damage). This showed that MMP9 is activated by $S$-nitrosylation of a cysteine residue followed by further oxidation to a sulphinic- or sulphonic-acid derivative. This latter step is particularly interesting, as it is irreversible, which would explain the permanent pathophysiological activation of
THERAPEUTIC PROTEINS

\section{High affinity good, lower affinity better}

When proteins bind their cognate receptors on the cell surface, signalling cascades are set in motion. The cytokine granulocyte colonystimulating factor (GCSF) stimulates the proliferation of certain immune cells and is used to treat cancer patients who have low levels of white blood cells as a result of cytotoxic drugs. Unfortunately, the value of such therapeutic proteins is limited because of rapid clearance by receptor-mediated endocytosis and consequent protein degradation. In the September issue of Nature Biotechnology, scientists from the Massachussetts Institute of Technology (MIT) and Amgen used computer modelling and protein chemistry to engineer GCSF variants with lower receptor affinity that are as biologically potent as wild-type GCSF, but remain active for longer.

Activation of a cytokine receptor causes internalization of the receptor-ligand complex by the cell and results in delivery of the complex to acidified endosomal compartments. At this point, the cytokine ligand is either sent to lysosomal organelles, where the protein is degraded, or it is routed to recycling vesicles, which transport the cytokine out of the cell. What determines the fate of the cytokine-receptor complex in the endosome? Evidence indicates that when the receptor-ligand complex dissociates in the endosome, both the ligand and receptor are more likely to be recycled to the cell surface. Recycling preserves the structural integrity and biological activity of the ligand, and leads to an increase in the amplitude of the signal from a given concentration of ligand. Sarkar et al. postulated that if they could increase the recycling of GCSF and its receptor without disrupting the ligand-receptor complex at the cell surface, the efficacy and potency of GCSF could be improved. Exploiting the $\mathrm{pH}$ difference in the endosome compared with the cell surface, the authors set out to create a cytokine that might have lower receptor affinity at a more acidic $\mathrm{pH}$.

Using the solved crystal structure of the interactions between GCSF and its receptor, a series of sites were identified at the ligand-receptor interface where the presence of a positive charge would destabilize the complex. Introducing histidine residues at these sites by site-directed mutagenesis resulted in a neutral charge at the cell surface and a positive charge in the endosome.
This allowed $\mathrm{pH}$-dependent modulation of the affinity of the complex, so that the GCSF variants bound to the receptor at the cell surface with equal affinity to that of the wild type, but with much lower affinity at the lower endosomal $\mathrm{pH}$. As the authors had predicted, in vitro assays showed that the variants were depleted less quickly from the growth medium and were more effective in promoting cell proliferation.

It is not yet clear whether the surface histidine mutations alter the antigenic effects of the protein, or its stability, and these engineered GCSF variants still need to be tested in an animal model. However, these variants show the validity of the approach, which could be widely applied to other protein ligands, whose efficacy is enhanced by increasing recycling back to the cell surface.

Melanie Brazil

(9) References and links

original RESEARCH PAPER Sarkar, C. A. et al. Rational cytokine design for increased lifetime and enhanced potency using pH-activated "histidine switching". Nature Biotechnol. 20, 908-913 (2002)

FURTHER READING Kangas, E. \& Tidor, B. Optimizing electrostatic affinity in ligand-receptor binding: theory, computuation, and ligand properties. J. Chem. Phys. 109, 7522-7545 (1998) | Lauffenburger, D. A., Fallon, E. M. \& Haugh, J. M. Scratching the (cell) surface: cytokine engineering for improved ligand/receptor trafficking properties. Chem. Biol. 5, R257-R263 (1998) WEB SITES

Lauffenberger's laboratory: http://web.mit.edu/cbe/dallab/ Tidor's laboratory: http://web.mit.edu/tidor/ 